\title{
Increasing Electrical Muscle Stimulation's Dexterity by means of Back of the Hand Actuation
}

\author{
Akifumi Takahashi \\ The University of Chicago, USA; The University of \\ Electro-Communications, Japan \\ a.takahashi@kaji-lab.jp \\ Hiroyuki Kajimoto \\ The University of Electro-Communications, Japan \\ kajimoto@kaji-lab.jp
}
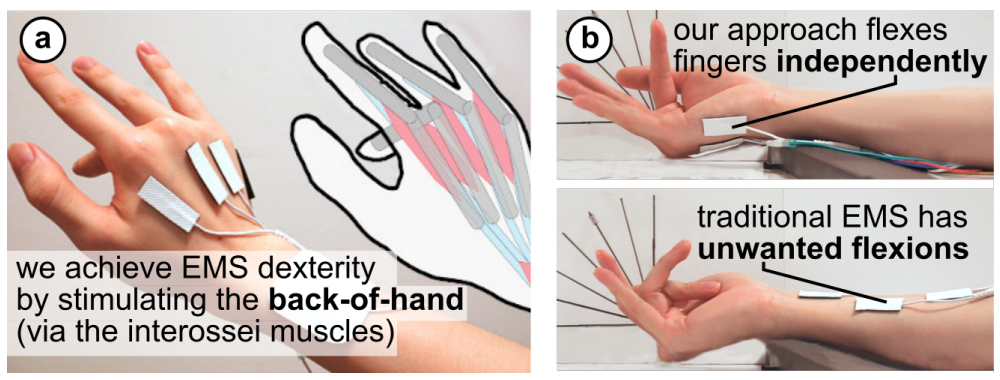

Jas Brooks

The University of Chicago, USA

jasbrooks@uchicago.edu

Pedro Lopes

The University of Chicago, USA

pedrolopes@uchicago.edu

Figure 1: We improved the dexterity of the finger flexion produced by interactive devices based on electrical muscle stimulation (EMS). The key to achieve it is that we discovered a new electrode layout in the back of the hand. Instead of the existing EMS electrode placement, which flexes the fingers via the flexor muscles in the forearm, we stimulate the interossei/lumbricals muscles in the palm. Our technique allows EMS to achieve greater dexterity around the metacarpophalangeal joints (MCP), which we demonstrate in a series of applications, such as (c) a two-stroke drum roll or (d) a barred guitar fret. These examples, as with all others in our paper, were previously impossible with existing EMS electrode layouts.

\begin{abstract}
We propose a technique that allows an unprecedented level of dexterity in electrical muscle stimulation (EMS), i.e., it allows interactive EMS-based devices to flex the user's fingers independently of each other. EMS is a promising technique for force feedback because of its small form factor when compared to mechanical actuators. However, the current EMS approach to flexing the user's fingers (i.e., attaching electrodes to the base of the forearm, where finger muscles anchor) is limited by its inability to flex a target finger's metacarpophalangeal (MCP) joint independently of the other fingers. In other words, current EMS devices cannot flex one finger alone, they always induce unwanted actuation to adjacent fingers. To tackle the lack of dexterity, we propose and validate a new electrode layout that places the electrodes on the back of the hand, where they stimulate the interossei/lumbricals muscles in the palm, which have never received attention with regards to EMS. In our user study, we found that our technique offers four key benefits
\end{abstract}

This work is licensed under a Creative Commons Attribution-Share Alike International 4.0 License.

CHI '21, May 08-13, 2021, Yokohama, Japan

(c) 2021 Copyright held by the owner/author(s).

ACM ISBN 978-1-4503-8096-6/21/05.

https://doi.org/10.1145/3411764.3445761 when compared to existing EMS electrode layouts: our technique (1) flexes all four fingers around the MCP joint more independently; (2) has less unwanted flexion of other joints (such as the proximal interphalangeal joint); (3) is more robust to wrist rotations; and (4) reduces calibration time. Therefore, our EMS technique enables applications for interactive EMS systems that require a level of flexion dexterity not available until now. We demonstrate the improved dexterity with four example applications: three musical instrumental tutorials (piano, drum, and guitar) and a VR application that renders force feedback in individual fingers while manipulating a yo-yo.

\section{CCS CONCEPTS}

- CSS CONCEPTS; • Human-centered computing; • Human computer interaction (HCI); • Interaction devices; • Haptic devices;

\section{KEYWORDS}

Electrical muscle stimulation, EMS, dexterity, haptic actuation

\section{ACM Reference Format:}

Akifumi Takahashi, Jas Brooks, Hiroyuki Kajimoto, and Pedro Lopes. 2021. Increasing Electrical Muscle Stimulation's Dexterity by means of Back of the Hand Actuation. In CHI Conference on Human Factors in Computing Systems (CHI '21), May 08-13, 2021, Yokohama, Japan. ACM, New York, NY, USA, 12 pages. https://doi.org/10.1145/3411764.3445761 


\section{INTRODUCTION}

EMS is a promising technique for force-feedback \& haptic-actuation because of its small form factor when compared to mechanical actuators (e.g., exoskeletons [1] and so forth). Therefore, EMS has been recently used to enable a wide range of haptic applications, such as: moving a user's wrist to tap to a beat $[2,3]$, teaching users how to manipulate objects they have never seen before [4], communicating information eyes-free [5] or even controlling the user's hand, which is holding a pen, to act as a plotter device [6].

However, all these aforementioned EMS systems, and all others in the literature, are limited in that they either do not actuate the user's fingers (mostly only the wrist) or actuate the user's fingers very coarsely and not independently of each other, i.e., in most of these systems a particular target finger is flexed by means of EMS and other fingers start flexing as well, causing unwanted actuation and lack of dexterity-we denote this as the dexterity of the movements/poses induced by EMS on the user, not the user's own dexterity. In fact, the lack of dexterity in EMS-induced finger flexions was well documented in the PossessedHand [7], a work that pioneered the use of EMS to build interactive devices. In this system, the authors found that they could not flex any finger around the metacarpophalangeal (MCP) joint independently of the other fingers. Although 10 years have passed since the PossessedHand, it still stands as the most dexterous finger flexions in all interactive EMS devices.

We identify the cause of EMS's lack of dexterity when inducing a finger flexion, pinning it down to the fact that virtually every researcher and practitioner realizes it by placing electrodes at the forearm. While this location intuitively makes sense, since all the finger flexor muscles are anchored there, it also is the cause for the lack of dexterity for three key reasons: (1) the flexor muscles in the forearm are densely packed, as they all meet at the elbow as a shared anchor; as such, attempting to flex a finger by means of electrodes attached to the skin causes currents to run also through an adjacent muscle, making other muscles move unwantedly; (2) the flexor muscles at the forearm are layered with finger flexors at one depth and wrist flexors at another; as such, attempting to flex a finger by means of electrodes attached to the skin often causes currents to run through the other layers as well, causing other muscles (e.g., wrist) to move unwantedly; (3) when we turn our wrist, the skin at the forearm rotates by a different amount compared to the muscles at the forearm; thus, whenever the wrist moves, the electrodes end up stimulating a new muscle, causing unwanted flexions.

To illustrate the importance of this problem, our literature review found that, in just the last 17 years of EMS research in HCI/Haptics, 54 publications [2-55] have used, tested, described, or proposed applications that use this exact electrode placement on the forearm to achieve finger flexions. All these systems, unfortunately, do not exhibit more dexterity in the finger flexions because they are limited by this electrode layout, which by now has become so "standard" it goes unquestioned by researchers.

To address the lack of dexterity in EMS, we discovered a novel electrode arrangement, depicted in Figure 1 (a), that robustly enables individual finger flexion. Our approach differs from the standard-EMS electrode arrangement in that we place the EMS electrodes on the back of the hand and instead stimulate the interossei/lumbricals muscles in the palm.

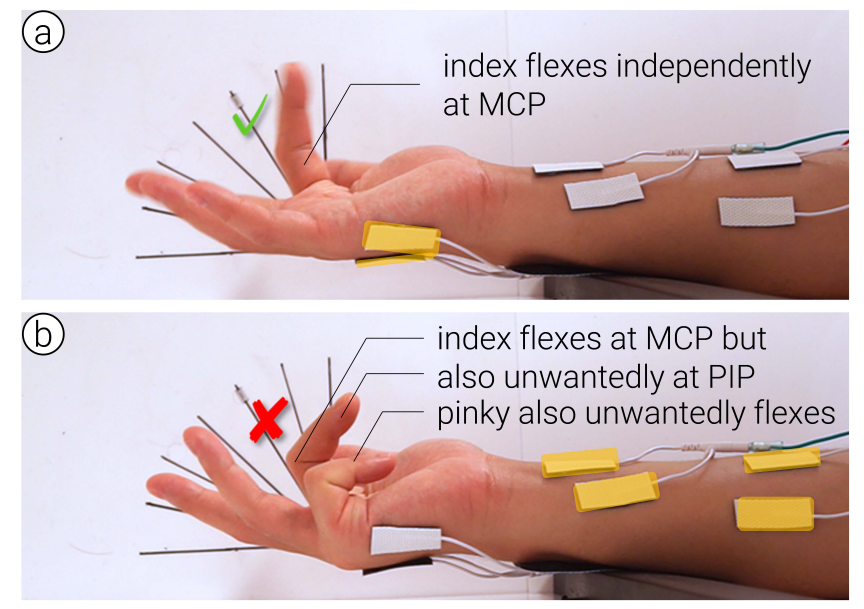

Figure 2: For this participant, (a) the index finger was independently flexed at the MCP joint by our back-of-hand stimulation, yet (b) using the standard-EMS stimulation, it induces several unwanted effects, e.g., flexion of other fingers or of the PIP joint.

Surprisingly, we found through a user study, that this new electrode arrangement provides four key benefits when compared to the standard-EMS arrangement: our technique (1) flexes all four fingers around the metacarpophalangeal joint (MCP) more independently; (2) has less unwanted flexion of other joints (such as the proximal interphalangeal joint); (3) is more robust to wrist rotations; and (4) reduces calibration time.

To visually illustrate the difference in dexterity achieved by our approach when compared to the standard-EMS approach, we depict in Figure 2 one striking example of the resulting actuation of the index finger of an exemplary participant from our User Study.

\section{RELATED WORK}

Our work builds primarily on the field of haptics, in particular electrical muscle stimulation. Also, to familiarize the reader with the kinematics of the hand, we present first an overview of its joint mechanisms and musculature.

\subsection{Human Hand Joints}

Most humans have five digits per hand, four fingers (index, middle, ring, and pinky), and a thumb. Each finger has three joints: distal interphalangeal (DIP), proximal interphalangeal (PIP), and metacarpophalangeal $(\mathrm{MCP})$ as ordered from the tip of the finger to the palm. In our paper, we focus on flexing the fingers around the MCP joint as well as minimizing concurrent unwanted flexions of the PIP joint.

\subsection{Motor-Based Finger Actuation (e.g., Exoskeleton Gloves)}

One way that researchers have long explored actuating the user's fingers to provide force feedback or guidance is by means of motorbased haptic gloves. Exoskeleton gloves are one example that successfully achieved dexterous manipulation by essentially adding 
an actuator (typically a motor with a pulley) to every finger's $\mathrm{MCP}$ and/or PIP joints. These motors connect the finger segments via mechanical linkages to achieve maximum force transmission when pulling against the palm (the base). While many exoskeleton designs exist, including soft or brake-based exoskeletons, the most dexterous devices tend to be rigid, such as the haptic workstation [56], which is one of the earliest fully-dexterous mechanical haptic devices for the hands. Other notable examples include cyber grasp [57], Sandoval-Gonzalez et al.'s exoskeleton [1], or dexmo [58], just to cite a few.

\subsection{Electrical Muscle Stimulation}

Electrical Muscle Stimulation (EMS) is a technique to induce muscle contractions by means of electrical impulses, typically via electrodes attached on the skin. EMS originated in the 1960s' medical rehabilitation as a means to restore lost motor functions [59]. Only more recently have researchers in HCI started to explore EMS with the pioneering uses of interactive EMS by Kruijff et al. for flexing the biceps as video-game feedback [60] and the aforementioned PossessedHand [7], which induced finger flexions and extensions.

While the aforementioned motor-based haptics are more precise than the resulting flexion quality induced by muscle stimulation, EMS is not without its advantages over its mechanical-counterpart. EMS systems are generally more wearable as their resulting formfactor is much smaller than motor-based devices [61]. As such, many researchers consider EMS as an ideal actuation platform for mobile haptics, such as force-feedback in VR [33], AR [32], or haptic instructions [11, 34, 43, 55].

However, when we zoom into each of these interactive devices based on EMS, we see that their actuation quality is coarse and imprecise. Most systems target simple joints, like the wrist, and only a smaller number, such as the PossessedHand, target moving fingers. More importantly, while the PossessedHand attempted to have their participants learn how to play a string-based instrument, the researchers found in their study that its electrode placement, which is now the de-facto "standard" in EMS, could only actuate three fingers independently around the PIP joint: index, middle, and ring. They found that EMS could not move any finger independently around the MCP joint. This is a critical limitation of today's EMS since it prevents building haptic applications that require any form of finger dexterity. Unsurprisingly, this is why applications such as "playing piano with EMS" are desirable, but, 10 years after the introduction of EMS at CHI, we still cannot realize it.

Some promising advances have been made to this end, such as Watanabe et al.'s [52] method to control the middle finger at the MCP and PIP joints by stimulating both its flexor and extensor. While they succeeded in controlling this finger, they did not create reliable actuations around the MCP joint for all fingers.

The reason why the independent flexion of fingers under EMS control has been so elusive is tied to the location that all these researchers place their electrodes at: the forearm.

\subsection{Muscle Arrangement for Flexors of MCP joint at the forearm ("standard-EMS")}

Figure 3 depicts the cross-section of the forearm. The key insight that drives the standard-EMS arrangement is that the finger flexor
(MCP) muscles are packed densely adjacent to one another and also layered one on top of each other inside the forearm. Therefore, researchers have been using this location in virtually any EMS system that achieves finger flexion, including usages outside of HCI such as in medicine [54, 55, 62].

To illustrate the wide-spread use of this electrode placement, we found that in just the last decades of EMS research in HCI/Haptics, 54 publications [2-55] have used, tested, described, or proposed applications that use this exact electrode placement on the forearm to achieve finger flexions. Following, this arrangement emerged as a de-facto "standard-EMS" placement. In fact, like all our community, we have used this arrangement extensively in our own EMS research in both HCI and neuroscience.

\section{OUR APPROACH FOR PRECISE FLEXOR ACTUATION: EMS ON THE BACK OF THE HAND}

We solve the lack of dexterity in EMS by discovering a new electrode arrangement. Instead of placing the electrodes at the base of the arm to target the finger flexors at that location, we stimulate the back of the hand, targeting the unexplored lumbricals/interossei. As we found in our empirical study, this results in more dexterity when flexing fingers around the MCP joint using EMS.

The key insight that enables our approach is depicted in Figure 4. Interestingly, the hand's dorsal side has no extensor muscles and most of the dorsal muscles are interossei and lumbricals muscles, which happen to be MCP finger flexors. Importantly, these muscles are close to the surface and spaced from each other, making them ideal targets for EMS activation by placing electrodes on the back of the hand. While this might appear as trivial in hindsight, it has been completely overlooked and presents an immediate and significant impact on the dexterity of any EMS-based interactive devices that use any type of finger flexions.

\section{BENEFITS, CONTRIBUTION AND LIMITATIONS}

Our key contribution is that we discovered a new electrode arrangement that enables any interactive device based on EMS finger flexion to actuate the user's fingers with more dexterity. Instead of placing the electrodes at the forearm, we stimulate the interossei/lumbricals at the back of the hand.

Our contribution was validated by a user study, providing a thorough characterization of the technique's accuracy. We found that our technique has four key benefits when compared to standardEMS placement: our technique (1) flexes all four fingers around the metacarpophalangeal joint more independently; (2) has less unwanted flexion of the PIP joints; (3) is more robust to wrist rotations, i.e., users can finally move their hands freely while EMS still flexes their fingers accurately; and (4) reduced calibration time.

Our approach is not without its limitations. (1) Like any EMS approach, it requires calibration prior to use, but we found that it reduces this calibration time. (2) It targets only the MCP flexor muscles, not the extensors or PIP flexor muscles. While the MCP independence that we achieve is key for many haptic applications, it is not the complete vocabulary for all hand-poses. We believe 


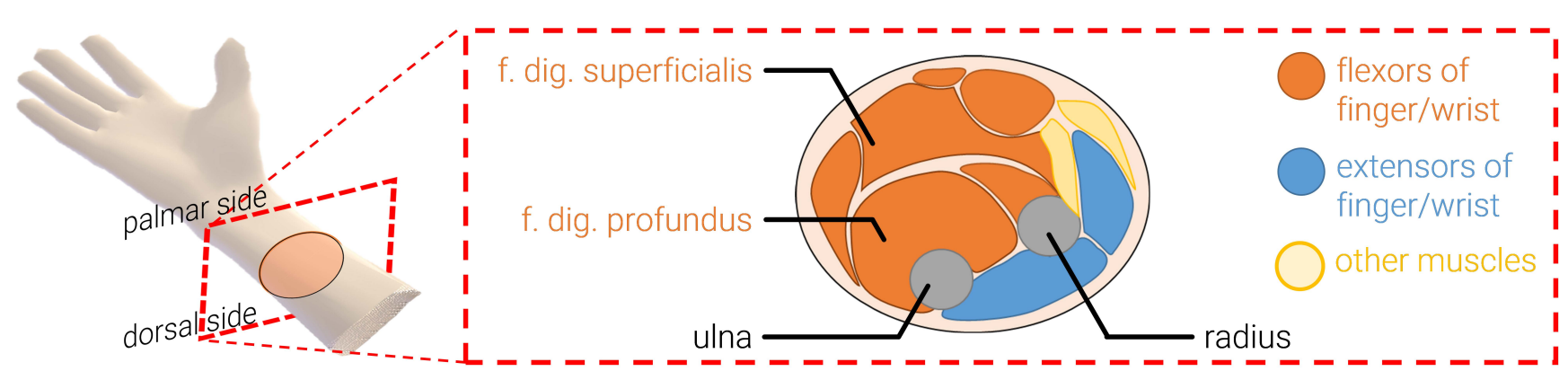

Figure 3: A model of cross-section muscle arrangement in the forearm and the hand, drawn from anatomy literature [63].

that the latter can be resolved by integrating both the standardEMS approach with our novel arrangement, thus stimulating both flexor and extensor around the MCP. Note that our approach cannot possibly target any extensor muscles since there are none in the palm.

\section{USER STUDY: INDEPENDENCE OF FINGER FLEXION DURING EMS ACTUATION (MCP JOINT)}

In our user study, we aimed to validate our key contribution, i.e., stimulating the back of the hand to achieve a higher level of dexterity than the standard EMS approach that stimulates the forearm flexors. As such, we measured the angles of participants' fingers (all four fingers, no thumb) when stimulated by means of either our back-of-hand EMS approach or the standard-EMS approach, which stimulates the finger flexion around the MCP joint via the flexor muscles at the base of the arm. By observing the fingers as they flex, one can extract the index-of-independence of each finger when actuated by means of EMS, i.e., how much this finger moves independently of the other. This is a physiological measure, used also in [64], to evaluate the independence of finger movements. This study was approved by our local Ethics Review Board (ID\#18044).

\subsection{Four Hypotheses}

We postulated four hypotheses for our experiment. For our main hypothesis (H1), we expected that our back-of-hand EMS approach would actuate each finger more independently of each other (i.e., minimize unwanted movement of adjacent fingers) than the standard-EMS approach. For our secondary hypothesis, (H2) we expected that our approach would lead to less unwanted movement on other joints, i.e., we expected that our approach would lead to less actuation of the PIP joints when targeting the MCP joints. For our tertiary hypothesis, (H3) we expected our approach to be more rotation invariant than the standard-EMS, i.e., we expected that back-of-hand EMS would actuate fingers more independently of each other even if the user's palm was rotated by an extreme 180 degrees (palm down). Lastly, we postulated (H4) that calibration time would be reduced for back-of-hand stimulation; this should occur as back-of-hand actuation targets the interossei/lumbrical muscles, which are not as heavily layered as the forearm flexor muscles targeted in the standard-EMS approach.

\subsection{Interface Conditions}

Each participant experienced our study in two distinct interface conditions: (1) back-of-hand EMS stimulation, in which the electrodes were applied using our novel layout by targeting the dorsal side of their hand; and, (2) standard-EMS, in which the electrodes are placed along the flexor muscles of the forearm (as often used in EMS research [2-55], but most canonically in Possessed Hand [7]). For either condition, we utilized the same EMS stimulator with the same stimulation pattern.

\subsection{Electrode Placement}

For our back-of-hand electrode placement, we utilized five electrodes: one $1 \times 3 \mathrm{~cm}$ electrode for each finger as the positive end,

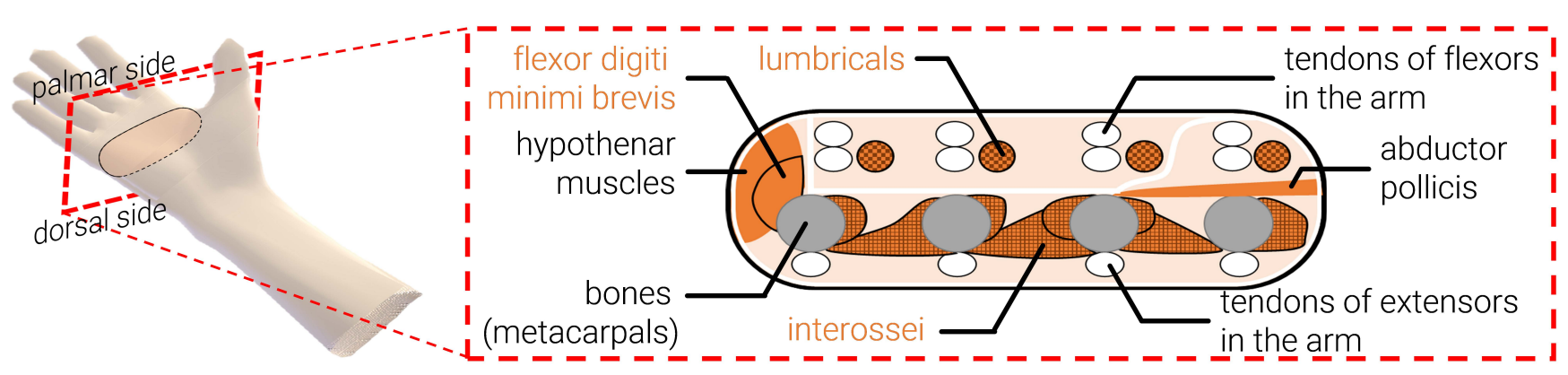

Figure 4: The key insight that enables our approach to achieve dexterity is that the lumbrical and interossei muscles, at the back of the user's hand, are more easily accessible to EMS than the standard forearm flexor muscles. 
which was placed alongside the respective finger's metacarpus (four total for four fingers), and one $3 \times 3 \mathrm{~cm}$ electrode as common ground, which was placed at the wrist.

For the standard-EMS electrode placement, we followed the placement described by Possessed Hand [7], selecting only the relevant electrodes for our comparison, which are the flexor electrodes. As such, we also place five electrodes: one $1 \times 3 \mathrm{~cm}$ electrode as the positive end, placed over the respective flexor muscle at the forearm (as in [7]), and one $5 \times 5 \mathrm{~cm}$ electrode as common ground, which was placed at the wrist. We follow a calibration procedure as described in Possessed Hand [7] and calibrate each finger movement one by one to achieve maximum independent motion by optimizing the placement for each user's arm anatomy.

\subsection{Apparatus}

We used a keyboard wrist rest so that participants rested their forearm comfortably during the trials. This minimized the effect of fatigue from unrelated muscles such as biceps, triceps, etc.

To capture the angles of both MCP and PIP joints accurately, we instrumented participants' hands with wearable inertial measurement units (with 6DOF sampled at $100 \mathrm{~Hz}$ ). We added one 9DOF IMU per finger joint and an additional one at the dorsal part of the hand for calibration and reference, totaling nine IMUs. We initially confirmed the accuracy of our IMU tracking system by comparing its accuracy when recording movements against a ground truth protractor (angle measurement). We recorded an accuracy of 5 degrees with a drift of $2 \% \mathrm{~min}$. Since our single trials last only for 4 seconds, this drift had virtually no impact, as our apparatus re-calibrated the IMU's drift prior to every trial.

Lastly, both our condition and the baseline used the same EMS stimulator (the same used in [65]) and the same stimulation waveform (biphasic waveform, with a pulse width of $200 \mu$ s for the positive phase, $100 \mu$ s rest period, and $200 \mu$ s for negative phase) at $50 \mathrm{~Hz}$. As common in EMS research, the intensity (current) was calibrated per-participant to ensure pain-free operation. Lastly, we filmed participants' fingers via a side-camera.

\subsection{Calibration}

Prior to the experiment, we calibrated the EMS for each participant. First, to determine the stimulation intensity (i.e., current, in $\mathrm{mA}$ ) we actuated the back-of-hand flexor of the participant's index finger. We started with an intensity of $0 \mathrm{~mA}$ and a pulse-width of 200 $\mu$ s (which we call zero point) and slowly increased the intensity in $1 \mathrm{~mA}$ steps until the participant's finger was fully flexed; this newly found intensity value was then fixed for all conditions and fingers and all further adjustments were made only by varying the pulse-width-this process effectively normalizes intensity values across multiple users, allowing us to compare intensity values by comparing the pulse-width required to actuate each finger (Figure 5). Then, after fixing the intensity for this participant, we adjusted the pulse-width (in $\mu$ s) to actuate all fingers in both conditions. In this step, we calibrated following a manual version of PossessedHand [7]: (1) attached electrodes to the target muscles following anatomical guide; (2) per electrode, started with low pulse-width and increased it step-by-step, confirming at each step with the participant that the stimulation was is pain-free; (3) repeated until no more flexion happens at the target finger or pain has been reached (the latter never occurred). Before starting the trials, we validated the calibration and recalibrated if needed, removing any confound in the order of each condition's calibration.

After calibrating all participants, we found an average current of $9.23 \mathrm{~mA}(\mathrm{SD}=2.3)$, and the average pulse-width, per finger and condition, is depicted in Figure 5. As observed, both conditions require very similar pulse-widths to flex the fingers.

\subsection{Task and Procedure}

Each participant performed a total of 48 trials: 2 conditions (backof-hand or standard-EMS) $\times 4$ fingers $\times 3$ repetitions $\times 2$ palm directions (up or down). Per trial, a 2 s stimulation was applied in a randomized order and the resulting pose of the four fingers was measured via video (from $1 \mathrm{~s}$ after the stimulation to $1 \mathrm{~s}$ after the end of a trial). We instructed participants to relax their hands before every single trial started.

\subsection{Participants}

We recruited nine participants (all self-identifying as male; all righthanded) from our local institution. Participants received a 1000 JPY compensation. Only four participants had previously experienced EMS.

\subsection{Metric: Independence Index}

Our study is based on the independence index (also known as I.I. in literature), a standard metric used to measure the amount of independent movement of a finger. The independence index denotes the ratio of movement between a finger's joint (measured in the angle of the joint's movement) to how much the other joints moved [64]. Thus, when considering only the MCP joint, the independent index of a target finger $i\left(I I_{i}\right)$ can be calculated as follows:

$$
I I_{i}=1-\frac{\frac{1}{3} \sum_{k \in \Gamma_{\mathrm{i}}}\left|A_{k}\right|}{\left|A_{i}\right|}, \text { where, } A_{k}=\int_{\mathrm{T}} \theta_{k} \mathrm{~d} t,
$$

Here, $i$ denotes a stimulated (target) finger and $\Gamma_{\mathrm{i}}$ is a set of notstimulated fingers. An independence index of 0 or lower signifies that moving the target finger caused movement in the other fingers instead of independent movement of the target finger itself. Conversely, an index closer to 1 signifies more independence, with a perfect " 1 " depicting a complete independent movement of the target finger (with no unwanted movements from other fingers). However, it is critical to note that, the human hand's biomechanics do not exhibit fully independent fingers. As experienced in daily life, many fingers bend when other fingers move. Lang et al. measured an independence index of 0.84 for passive finger flexions around the MCP joint when a participant's finger was bent passively by a motor to extract its natural limits [64]. For the sake of visual clarity, we depicted this value of 0.84 as a dashed line in all our charts (annotated as "maximum voluntary independence").

\subsection{Results}

We analyzed the effects of interface condition, palm direction, and target finger on the independence index scores, and their possible interactions, by a model of multivariate analysis of variance (MANOVA), followed by four separated univariate three-way ANOVA analyses. 


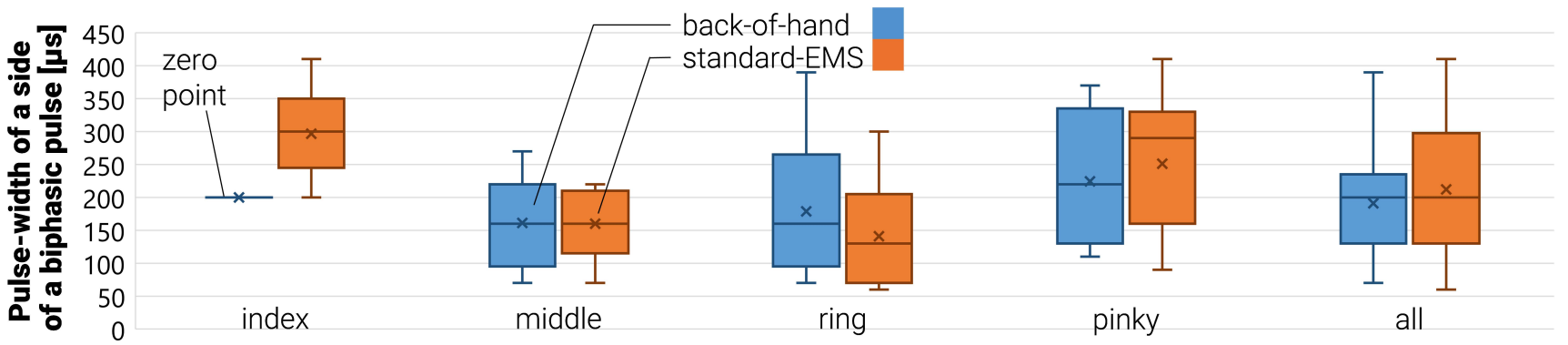

Figure 5: Resulting pulse-width after calibration for all fingers in both conditions (index finger as zero point; see calibration).

The MANOVA model included the aforementioned three factors (interface condition, palm direction, target finger) as independent variables and the MCP independence index and the ratio of PIP-toMCP movement as dependent variables. This MANOVA analysis showed a significant main effect of interface condition [Pillai's trace $0.181, F(2,127)=14.1, p<0.001]$. Further, a significant effect emerged for palm direction [Pillai's trace $0.0649, \mathrm{~F}(2,127)=4.41, \mathrm{p}<0.05$ ]. The three-way ANOVA analyses showed similar significant effects of the interface condition on the MCP independence index $[\mathrm{F}(1,128)=20.5$, $\mathrm{p}<0.001]$ and the ratio of PIP-to-MCP movement $[\mathrm{F}(1,128)=26.1$, $\mathrm{p}<0.001]$.

Figure 6 depicts our results with respect to the differences between the dexterity of stimulating the fingers around the MCP joint with back-of-hand compared to standard-EMS, as measured by the independence index. Taken together, our previously reported main effect of interface conditions [Pillai's trace 0.181, $\mathrm{F}(2,127)=14.1$, $\mathrm{p}<0.001]$ and the significant effect of the conditions on the MCP independence index $[\mathrm{F}(1,128)=20.5, \mathrm{p}<0.001]$ allow us to reject the null hypothesis (that there is no difference between conditions on MCP independence index scores) and support our H1. In fact, as depicted in Figure 6, we found that the index of independence averaged for all fingers was higher when actuated via back-ofhand EMS $(M=0.62 ; S D=0.21)$; compared to standard-EMS $(M=$ $0.18 ; \mathrm{SD}=0.81$ ); this suggests that overall actuation by means of back-of-hand EMS is more dexterous than the standard-EMS practice.
In particular, we measured an increased independence index in all four fingers: (1) the index finger was actuated with an average independence index of $0.62(\mathrm{SD}=0.33)$ with back-of-hand and an average of $-0.08(\mathrm{SD}=1.00)$ with standard-EMS; $(2)$ the middle finger was actuated with an average independence index of 0.563 $(\mathrm{SD}=0.14)$ with back-of-hand and an average of $0.34(\mathrm{SD}=0.37)$ with standard-EMS; (3) the ring finger was actuated with an average independence index of $0.59(\mathrm{SD}=0.16)$ with back-of-hand and an average of $0.33(\mathrm{SD}=0.34)$ with standard-EMS; and, lastly, (4) the pinky finger was actuated with an average independence index of $0.72(\mathrm{SD}=0.14)$ with back-of-hand and an average of 0.14 $(\mathrm{SD}=1.16)$ with standard-EMS.

Next, we analyze our second hypothesis, in which we postulated that our back-of-hand would exhibit less unwanted actuation of the PIP joint than standard-EMS. Our previously reported significant effect of the ratio of PIP-to-MCP movement $[\mathrm{F}(1,128)=26.1$, $\mathrm{p}<0.001$ ] allows us to reject the null hypothesis and support our H2. In fact, as depicted in Figure 7, we found that there were significantly more unwanted movements of the PIP joints when the participants' fingers were flexed using st andard-EMS $(M=0.83$, $\mathrm{SD}=0.48)$ than with back-of-hand EMS $(\mathrm{M}=0.41, \mathrm{SD}=0.19)$. In particular: (1) when actuating the index finger we observed a ratio of PIP movements with an average of $0.65(\mathrm{SD}=0.37)$ with standardEMS compared to a lower average of $0.37(\mathrm{SD}=0.14)$ with back-ofhand; similarly, we observed this trend on remainder fingers as depicted in Figure 7.

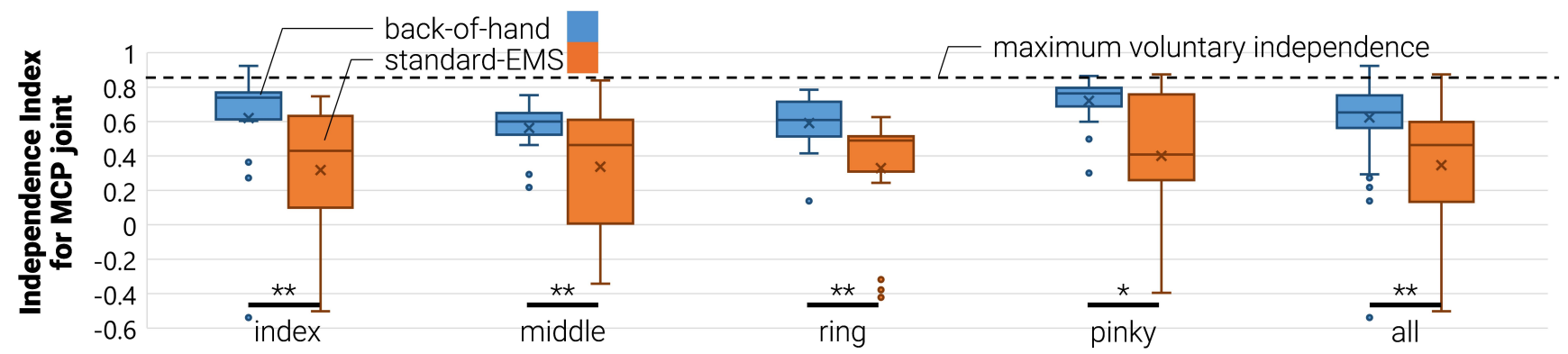

Figure 6: Measured index of independence when actuating each of the participants' fingers (index, middle, ring, pinky) using either back-of-hand EMS or standard-EMS; last box-plot depicts averages for all fingers. There are four outliers trimmed out of this plot for visualization clarity (for standard-EMS. $-1.64,-2.70,1.95$ in the index, -4.23 in the pinky). 


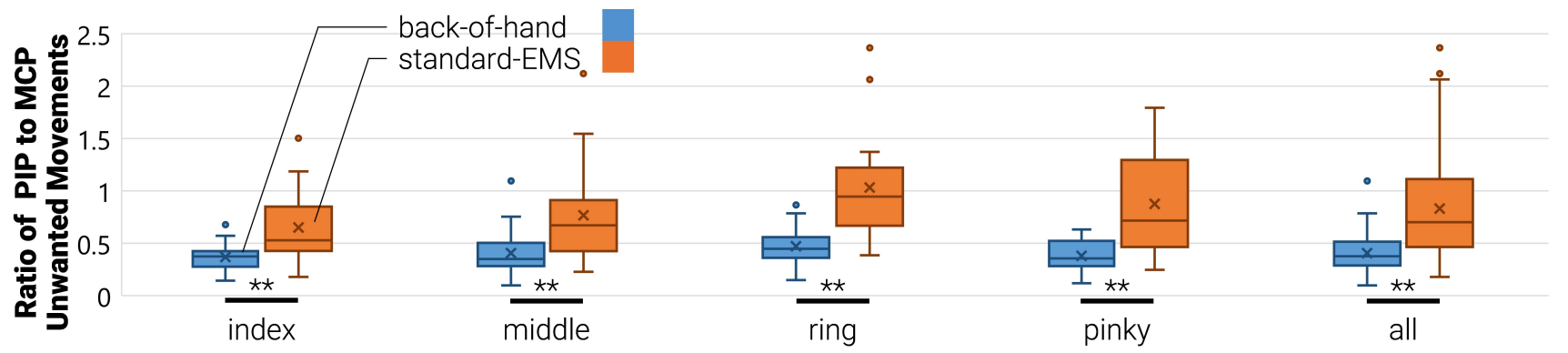

Figure 7: Measured ratio of unwanted PIP movements when flexing each of the participants' fingers around the MCP joint (index, middle, ring, pinky) using either back-of-hand EMS or standard-EMS.

Next, we analyze our third hypothesis, in which we postulated that our back-of-hand would allow for more independent finger flexions under extreme arm rotations than standard-EMS. Our previously reported significant effect of palm direction [Pillai's trace $0.0649, F(2,127)=4.41, p<0.05]$ allow us to reject the null hypothesis (that there is no difference between the interface conditions regarding palm direction); thus, there is a difference in these conditions, which we analyze next. In fact, as depicted in Figure 8, we found that the independence of standard-EMS decreased significantly as the participants' palms were simply turned down (drastic drop from $M=0.31, S D=0.45$ to $M=0.055, S D=1.04$ ) when compared to back-of-hand (a slight decrease from $M=0.65, S D=0.16$ to $M=0.60, S D=0.25)$. This supports our $H 3$. In particular, the middle finger illustrates the negative impact that the palm rotation had on the performance of the standard-EMS (decreasing from $\mathrm{M}=0.40, \mathrm{SD}=0.29$ to $\mathrm{M}=0.34, \mathrm{SD}=0.35$ ), but barely any impact in back-of-hand $(\mathrm{M}=0.57, \mathrm{SD}=0.13$ and similarly $\mathrm{M}=0.54, \mathrm{SD}=0.16)$. Moreover, as depicted in Figure 8 this trend is similar for the index finger.

As expected, the ring was the outlier, relatively stable in both conditions with minor improvement in independence in back-ofhand. This was expected because the ring is anatomically an easily accessible muscle. In fact, this is why a number of interactive EMS devices actuate precisely this finger, for instance in [24], authors state "we could robustly actuate it without any parasitical motion of neighboring muscles".

Finally, for the pinky finger, we observed an improvement for back-of-hand as the palm turned.

Last, we analyze our fourth hypothesis, in which we postulated that our back-of-hand would require less calibration time than standard-EMS. We found, by means of an independent $t$-test, a significant difference between the time to calibrate a participant per conditions $(p<0.01)$. This confirms our H4. In fact, depicted in Figure 9, we found that back-of-hand calibration is significantly quicker $(M=6.02, S D=2.59)$ than standard-EMS $(M=15.6, S D=7.9)$; note that we used the same calibration procedure for both and that we have far more years of experience with the standard-EMS calibration, having it performed thousands of times.

\subsection{Discussion}

Our results demonstrate that our method provides interactivesystems based on EMS with more independent actuation for all fingers around the MCP joint, when compared to the standard-EMS method. We now discuss our findings in detail, organized in five topics: (1) finger independence on MCP flexion; (2) minimizing unwanted actuation of other joints (e.g., PIP); (3) robustness to wrist rotations; and, (4) reduction of calibration time. Finally, we note that

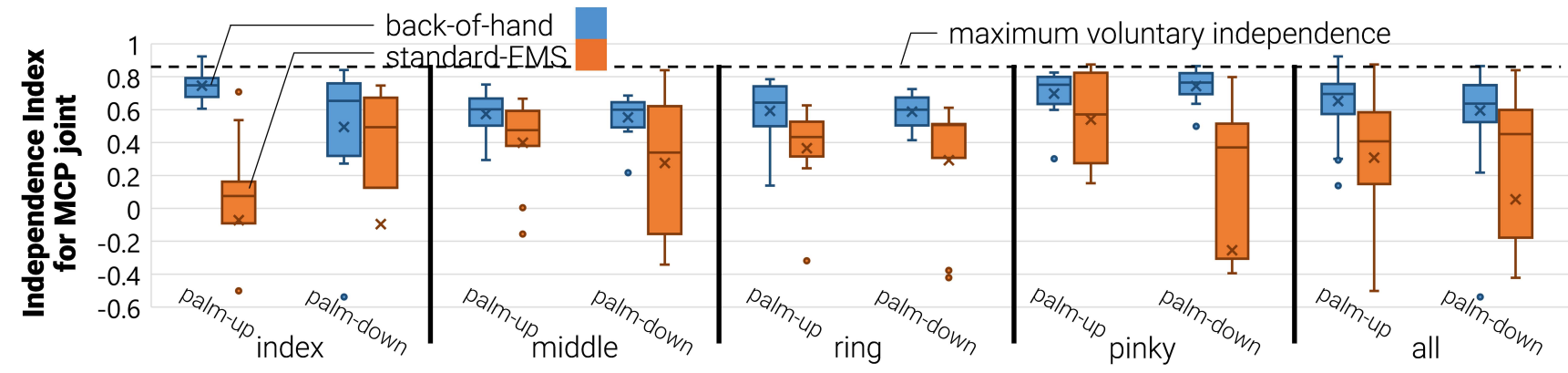

Figure 8: Measured index of independence when flexing each of the participants' fingers (index, middle, ring, pinky) in two different arm direction (palm-up or palm down) using either back-of-hand EMS or standard-EMS. There are four outliers trimmed out of this plot for visualization sake (for standard-EMS, one point (-1.64) in Index-Palm-Up, two points (-2.70 and 1.95) in Index-Palm-Down, one point (-4.23) in Pinky-Palm-Down). 


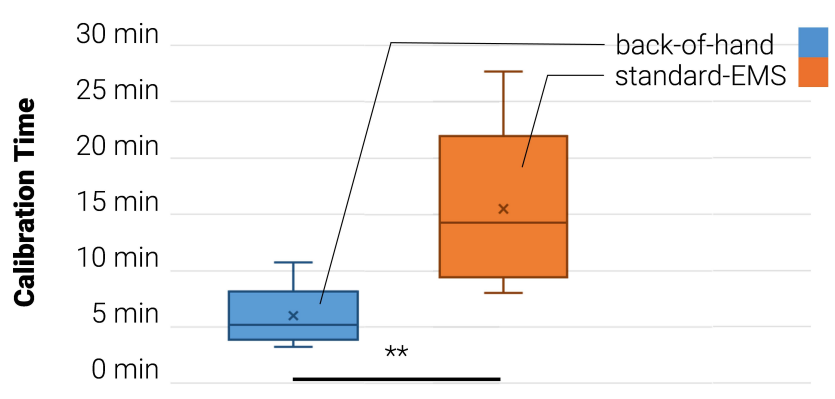

Figure 9: Calibration time using either back-of-hand EMS or standard-EMS.

with our sample size $(\mathrm{N}=9)$ our findings should not be generalized to variations in anatomy that might occur in different subjects.

5.10.1 Finger Independence During EMS Flexion Around the MCP Joint. Our main finding was that stimulating the muscles at the back of the hand results in more dexterous actuation of the flexor muscles, i.e., fingers flex independently of each other. As we demonstrate in our applications, this is critical to enable scenarios that depict realistic grasp poses. None of these are currently possible with the standard-EMS approach of stimulating the flexor muscles via the forearm. Our results for the finger independence during EMS actuation, depicted in Figure 6, demonstrated two key improvements: (1) flexion for all four fingers (index, middle, ring, and pinky) around the MCP joint improved in independence on average by $79.9 \%$ and, moreover, (2) the amount of standard deviation was reduced, suggesting our back-of-hand actuation is also more robust over multiple repetitions. To give the reader a visual illustration of the improved quality of the achieved dexterity, we selected a few examples from the study, which we depict in Figure 10.

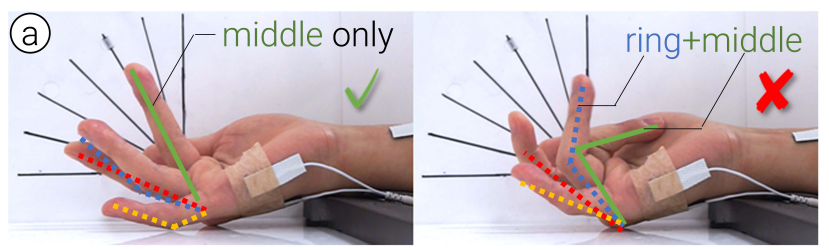

target: middle finger

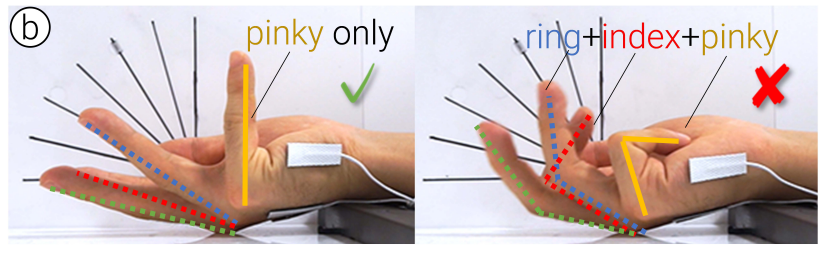

target: pinky finger

Figure 10: Examples from our study of EMS finger flexions targeted at the MCP joint: (a) middle finger a nd (b) pinky finger. " $\checkmark$ " denotes a correct finger flexion by back-ofhand, while " $X$ " denotes an incorrect finger flexion by standard-EMS.

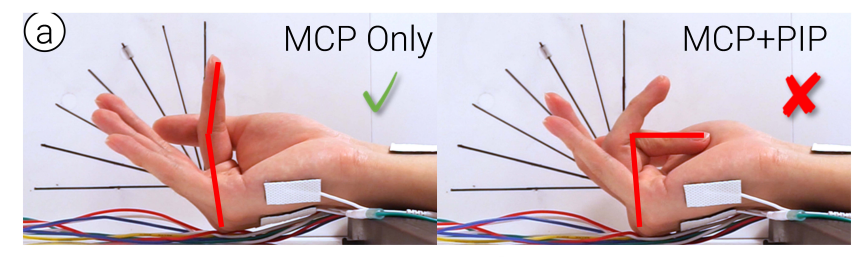

target: index finger

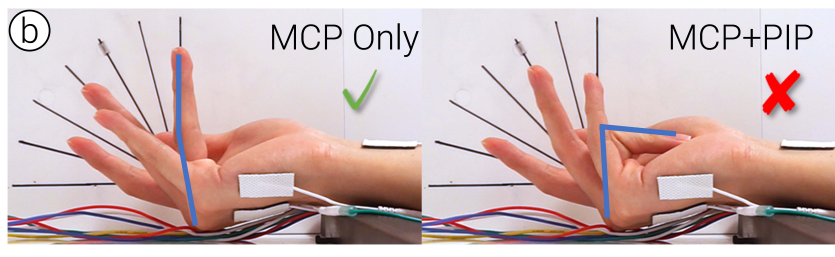

target: ring finger

Figure 11: Examples from our study of EMS finger flexions targeted at the MCP joint: (a) middle finger and (b) ring finger. " $\checkmark$ " denotes a correct finger $\mathrm{fl}$ exion by back-of-hand, while " $X$ " denotes an i ncorrect finger flexion by standardEMS.

5.10.2 Minimizing Unwanted Actuation of the PIP joint. One main limitation that Tamaki et al. discussed in PossessedHand [7] is that when actuating the MCP joint via the standard-EMS electrode position (base of the forearm) it induces unwanted flexions of the PIP joints. This is a showstopper for haptic applications that require finger dexterity, e.g., playing musical instruments, etc. Fortunately, we found that our technique significantly minimized the amount of unwanted flexion at the PIP joint, with an average reduction of $-51.2 \%$. To give the reader a visual illustration of the minimization of the unwanted movements of the PIP joint, we selected a few examples from the study, which we depict in Figure 11.

5.10.3 Robustness to Wrist Rotations. One critical limitation of EMS systems that actuate the fingers is that, when the user turns their palm, the actuation becomes imprecise for any finger flexions; this was well-documented and discussed in Affordance++ [4] and PossessedHand [7]. This happens because when the human arm rotates to turn the palm upside down, our skin (where the electrodes are attached) moves independently from the muscle structure. As such, when the hand rotates, the electrodes are displaced and then actuate the "wrong" portion of the flexor muscles. This is a major obstacle for applications where users move their hands, including virtually any practical application of EMS. Our finding was that our technique significantly improved the robustness of the flexion of the MCP joint during palm rotations, i.e., the actuation had a similar performance when the palm was up or down. To give the reader a visual illustration of the improvements when the palm is rotated, we selected a few examples from the study, which we depict in Figure 12.

5.10.4 Reduction in Calibration Time. One key limitation of any EMS system (ours included) is that they require calibration prior to use. This is well documented in the early works that pioneered the use of EMS in interactive systems, such as Kruiff et al. [60] and PossessedHand [7]. Our finding was that our technique significantly minimized the calibration time to one third of the time needed 

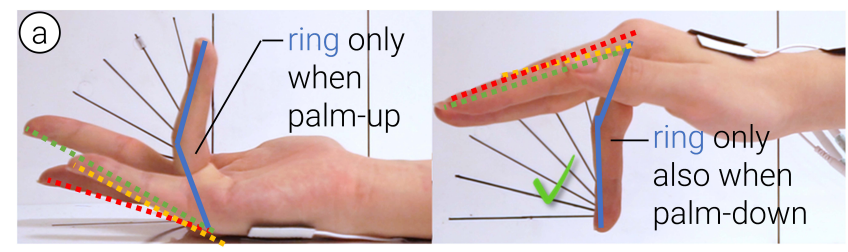

target: ring finger

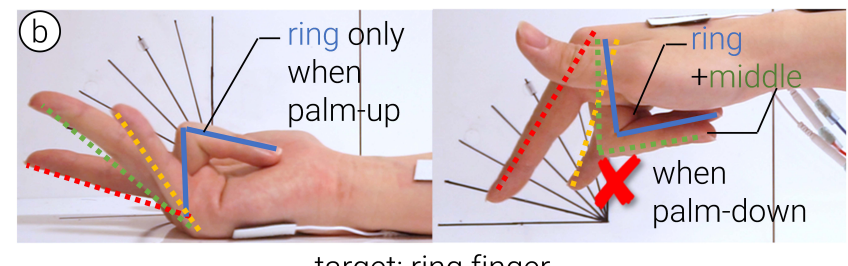

target: ring finger

Figure 12: Examples from our study of EMS ring finger flexions targeted at the MCP joint while rotating the palm (up or down). " $\checkmark$ " denotes a correct finger flexion by back-of-hand in both palm up or down, while " $X$ " denotes an i ncorrect finger flexion by $s$ tandard-EMS that dramatically decreased in quality when the palm was rotated down.

for standard-EMS. This is most likely caused by the fact that the muscles that our technique purposely targets are not as densely packed nor layered, which is the case for the finger flexors in the forearm. It is important to note that, in fact, the experimenters had more years of experience with the standard-EMS electrode layout, having performed thousands of standard-EMS calibrations for studies in $\mathrm{HCI}$ and neuroscience as well as demos at conferences. Unsurprisingly, even though we were very unfamiliar with the back-of-the-hand layout, it was significantly e asier t o calibrate, which explains the reduced calibration time. We believe this result is important not only for engineering interactive systems with significantly less calibration effort, but especially for anyone using EMS in both HCI, neuroscience, and rehabilitation.

\section{EXAMPLES OF DEXTROUS HAPTIC APPLICATIONS ENABLED BY OUR TECHNIQUE}

Now that we validated that our back-of-hand EMS approach affords more dexterity by allowing us to flex fingers more independently, we explore some of the applications that our contribution enables. Note that all these applications are novel in that they are simply not possible with the standard EMS approach because all of these require individual finger actuation with significant precision. For instance, while researchers have used EMS for drumming by coarsely moving the user's wrist or biceps up/down, we demonstrate how to drum using a professional drumming technique, the double stroke roll, which requires individual control of the ring finger.

\subsection{Actuating Double-Stroke Drum Rolls with EMS}

After drummers spend some time acquainting themselves with the basic stroke (hitting the drum pad with one swift motion of their wrist) they start exploring how to drum faster. This is typically
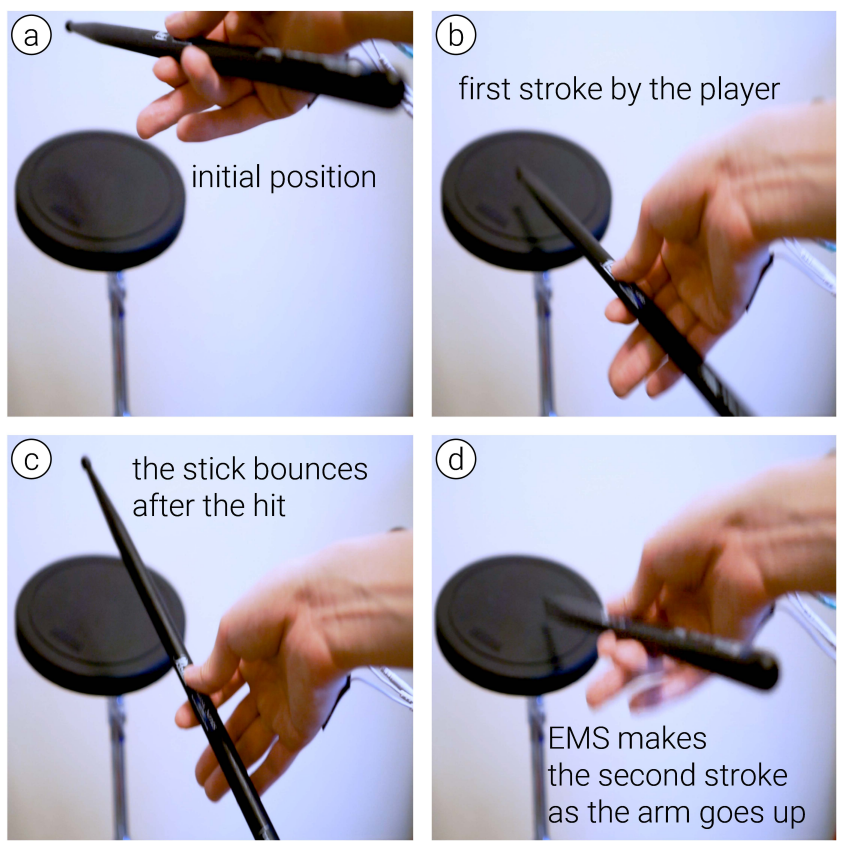

Figure 13: This drummer is being actuated by means of EMS to perform a double-stroke roll, a movement that requires significant finger independence (a quick finger flexion of the ring finger around the $\mathrm{MCP}$ joint) to realize.

achieved with a technique called double-stroke drum roll (Figure 13). Unlike the basic stroke, double-stroke rolls require a high level of dexterity, in which the motion of the second stroke is entirely driven by a very precise movement of the MCP joint alone of the ring finger; this is used by drummers using the socalled "French grip" to grab the drumstick. We implemented a simple haptic application, depicted in Figure 13, which actuates users in performing the two-stroke roll.

6.1.1 Actuating Fingers Independently via EMS to Play Piano Notes And Chords. Much like all applications in our paper, this is a longsought application in EMS that was never realized due to the lack of dexterity. However, we can now realize it via the back of the hand stimulation. In Figure 14, we depict a user who is being actuated to (a) play a simple one-handed piano melody with EMS finger flexions around the MCP joint for each finger; and, (b) play a perfect fifth interval by having the EMS flex their fingers to correctly play two notes at the same time. While our technique provides the first step towards EMS for piano playing by flexing the user's fingers around the MCP joint, it does not actuate all finger joints required to play piano in its full expression.

6.1.2 Actuating Fingers Independently To Play Barred Frets On The Guitar. For playing the guitar, an important skill is to press all strings at a fret with the same finger (usually the index finger), which is required to make a barre chord-we chose these types of chords as they require only MCP flexion, unlike generic chords that typically require flexion of other joints. Figure 15 depicts an example of our simple haptic application that actuates users how 

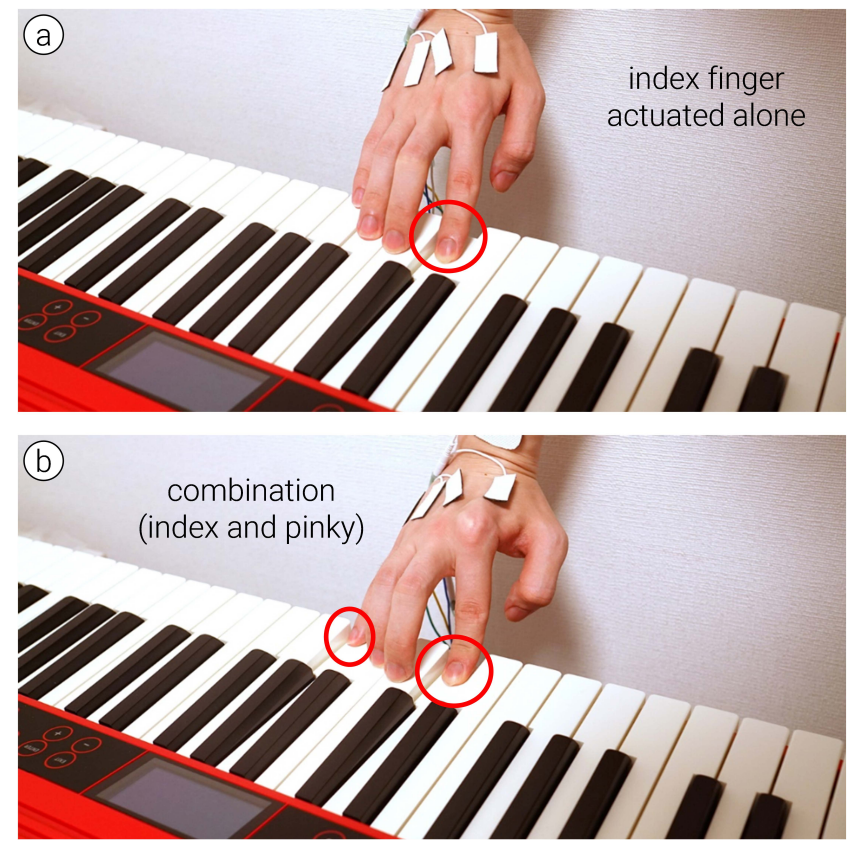

Figure 14: (a) With this newly achieved EMS dexterity, we can actuate a user to play simple piano melodies by having their fingers flexed, independently, by means of EMS. Also (b) we can assist a user in playing multiple notes and chords, such as this perfect fifth interval played, by means of EMS flexion of their index and pinky fingers at the MCP joint.

to make barred frets by independently flexing their fingers around the MCP joint.

6.1.3 Force Feedback for Individual Fingers in VR. While the previous applications used EMS to create motion guidance, our last application depicts an entirely different class: EMS to create force feedback. While EMS has been an increasingly popular technique to achieve wearable force feedback (e.g., [22, 32-34], there has not been any system that can stimulate each finger independently while retaining performance during wrist rotations, which is required by virtual reality (VR) applications where the user's hands move freely in space. In Figure 16, we exemplify this by means of a simple VR yo-yo application: (a) the user throws down a virtual yo-yo; then, (b) as the yo-yo's string hits its limit it pulls down on the user's middle finger, where the virtual yo-yo's string is knotted. Here, our back of the hand stimulation renders the force feedback of the yo-yo's pull by independently actuating a single finger, even under different wrist rotations caused by the user as they move their hands around to play with the yo-yo.

\section{CONCLUSIONS}

In this paper, we proposed, implemented, and tested a novel electrode layout that allows an unprecedented level of dexterity in electrical muscle stimulation (EMS), allowing interactive EMS-based devices to flex the user's fingers independently of each other. Our electrode layout places the electrodes on the back of the hand, where they stimulate the interossei/lumbricals muscles in the palm-a set
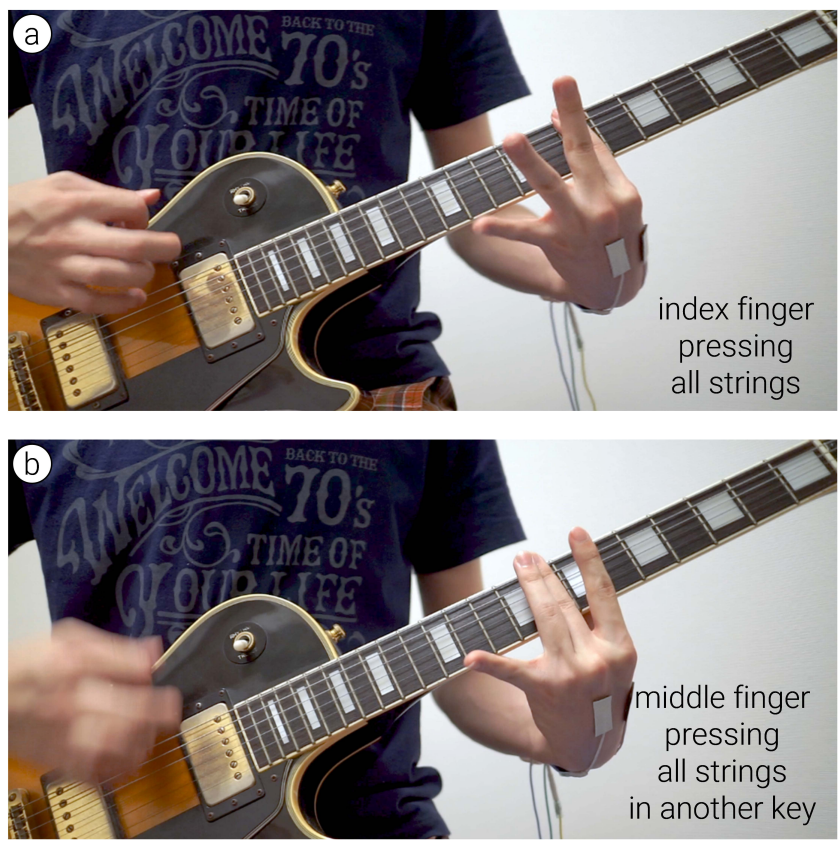

Figure 15: (a) Back-of-hand stimulation assists this user in performing a barred fret by flexing (a) their index finger and (b) their middle finger so that all strings at a fret are be pressed down completely.
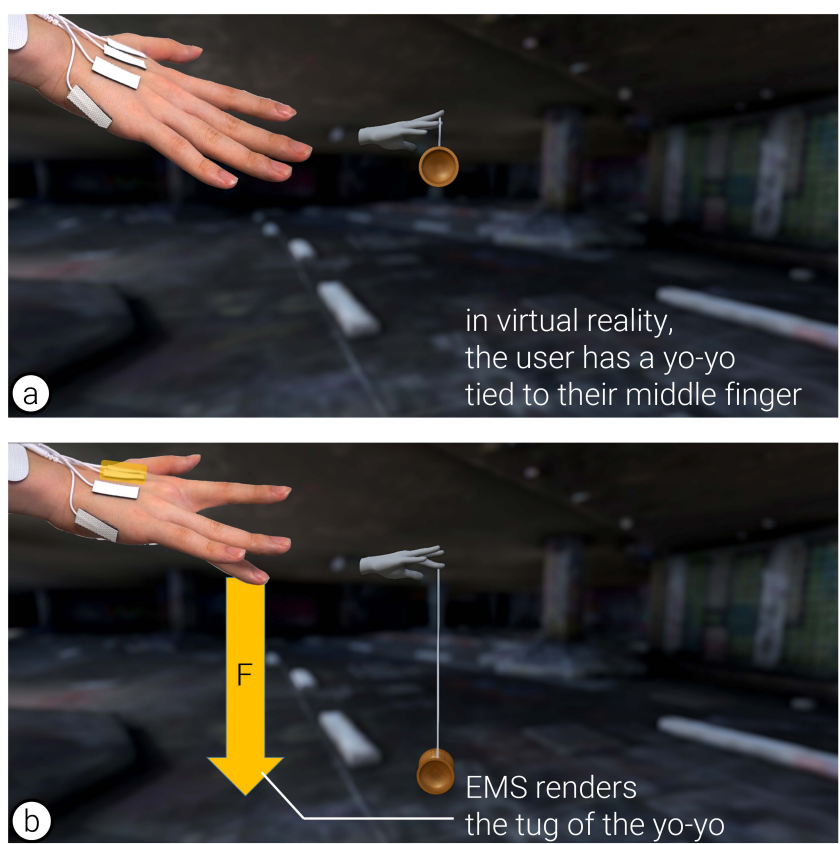

Figure 16: (a) The user has a virtual yo-yo in their hand with its string tied to the user's middle finger. (b) As the yo-yo is released, the yo-yo descends and pulls on the middle finger independently. The EMS from the back of the hand renders the yo-yo's force. 
of muscles that have been unexplored in EMS and that offer the following key improvements to the current EMS practice: (1) because these muscles are not as densely packed as the forearm flexors, our technique flexes all four fingers at the MCP joint more independently; (2) because these muscles are less densely layered than the forearm muscles, there is less unwanted flexion of other joints (such as from the proximal interphalangeal joint); (3) because these muscles are not subjected to forearm rotations, our technique is more robust to wrist rotations; and (4) because of all of the above, our technique reduced calibration time. We validated all these improvements by means of a user study.

To sum up, our EMS technique enables applications for interactive EMS systems that required a level of flexion dexterity unachievable until now. We demonstrate its dexterity with four example applications: three musical instrumental tutorials (piano, drum, and guitar), which require individual actuation of only a finger's MCP joint, and a VR application rendering force feedback for individual fingers when manipulating a yo-yo.

As for future work, we believe there are synergies to be found by combining the standard-EMS approach, which stimulates using the forearm muscles, with our back-of-hand approach, especially for the extensor muscles. Taken in combination, our technique and the previous approach might unlock the next level of dexterity and precision for EMS interfaces.

\section{ACKNOWLEDGMENTS}

Our research was supported by JSPS KAKENHI Grants (JP20J14306, JP18H04110). This work is supported in part by NSF grant 2047189. Any opinions, findings, and conclusions or recommendations expressed in this material are those of the authors and do not necessarily reflect the views of any funding agencies.

\section{REFERENCES}

[1] O. Sandoval-Gonzalez et al., "Design and Development of a Hand Exoskeleton Robot for Active and Passive Rehabilitation," Int. F. Adv. Robot. Syst., vol. 13, no. 2, p. 66, Mar. 2016, doi: 10.5772/62404.

[2] A. Ebisu, S. Hashizume, and Y. Ochiai, "Building a feedback loop between electrical stimulation and percussion learning," in ACM SIGGRAPH 2018 Studio, Vancouver, British Columbia, Canada, Aug. 2018, pp. 1-2, doi: 10.1145/3214822.3214824

[3] A. Ebisu, S. Hashizume, K. Suzuki, A. Ishii, M. Sakashita, and Y. Ochiai, "Stimulated percussions: method to control human for learning music by using electrical muscle stimulation," in Proceedings of the 8th Augmented Human International Conference on - $A H$ '17, Silicon Valley, California, 2017, pp. 1-5, doi: 10.1145/3041164.3041202.

[4] P. Lopes, P. Jonell, and P. Baudisch, "Affordance++: Allowing Objects to Communicate Dynamic Use," in Proceedings of the 33rd Annual ACM Conference on Human Factors in Computing Systems, New York, NY, USA, Apr. 2015, pp. 2515-2524, doi: 10.1145/2702123.2702128.

[5] P. Lopes, A. Ion, W. Mueller, D. Hoffmann, P. Jonell, and P. Baudisch, "Proprioceptive Interaction," in Proceedings of the 33rd Annual ACM Conference on Human Factors in Computing Systems, New York, NY, USA, Apr. 2015, pp. 939-948, doi: 10.1145/2702123.2702461.

[6] P. Lopes, D. Yüksel, F. Guimbretière, and P. Baudisch, "Muscle-plotter: An Interactive System based on Electrical Muscle Stimulation that Produces Spatial Output," in Proceedings of the 29th Annual Symposium on User Interface Software and Technology, Tokyo, Japan, Oct. 2016, pp. 207-217, doi: 10.1145/2984511.2984530.

[7] E. Tamaki, T. Miyaki, and J. Rekimoto, "PossessedHand: techniques for controlling human hands using electrical muscles stimuli," in Proceedings of the 2011 annual conference on Human factors in computing systems - CHI '11, Vancouver, BC, Canada, 2011, p. 543, doi: 10.1145/1978942.1979018.

[8] X. Bao, Y. Zhou, Y. Wang, J. Zhang, X. Lü, and Z. Wang, "Electrode placement on the forearm for selective stimulation of finger extension/flexion," PLOS ONE, vol. 13, no. 1, p. e0190936, Jan. 2018, doi: 10.1371/journal.pone.0190936.

[9] K. Chen, B. Zhang, and D. Zhang, "Master-Slave Gesture Learning System Based on Functional Electrical Stimulation," in Intelligent Robotics and Applications, vol.
8917, X. Zhang, H. Liu, Z. Chen, and N. Wang, Eds. Cham: Springer International Publishing, 2014, pp. 214-223.

[10] A. Colley, A. Leinonen, M.-T. Forsman, and J. Häkkilä, "EMS Painter: Co-creating Visual Art using Electrical Muscle Stimulation," in Proceedings of the Twelfth International Conference on Tangible, Embedded, and Embodied Interaction, New York, NY, USA, Mar. 2018, pp. 266-270, doi: 10.1145/3173225.3173279.

[11] T. Dingler, T. Goto, B. Tag, and K. Kunze, "EMS icons: conveying information by analogy to enhance communication through electrical muscle stimulation," in Proceedings of the 2017 ACM International Foint Conference on Pervasive and Ubiquitous Computing and Proceedings of the 2017 ACM International Symposium on Wearable Computers, New York, NY, USA, Sep. 2017, pp. 732-739, doi: $10.1145 / 3123024.3129275$.

[12] T. Duente, S. Schneegass, and M. Pfeiffer, "EMS in HCI: challenges and opportunities in actuating human bodies," in Proceedings of the 19th International Conference on Human-Computer Interaction with Mobile Devices and Services, New York, NY, USA, Sep. 2017, pp. 1-4, doi: 10.1145/3098279.3119920.

[13] T. Duente, J. Schulte, M. Pfeiffer, and M. Rohs, "MuscleIO: Muscle-Based Input and Output for Casual Notifications," Proc. ACM Interact. Mob. Wearable Ubiquitous Technol., vol. 2, no. 2, p. 64:1-64:21, Jul. 2018, doi: 10.1145/3214267.

[14] T. Duente, M. Pfeiffer, and M. Rohs, "On-skin technologies for muscle sensing and actuation," in Proceedings of the 2016 ACM International foint Conference on Pervasive and Ubiquitous Computing: Adjunct, New York, NY, USA, Sep. 2016, pp. 933-936, doi: 10.1145/2968219.2979136.

[15] T. Duente, M. Pfeiffer, and M. Rohs, "Zap++: a 20-channel electrical muscle stimulation system for fine-grained wearable force feedback," in Proceedings of the 19th International Conference on Human-Computer Interaction with Mobile Devices and Services, New York, NY, USA, Sep. 2017, pp. 1-13, doi: 10.1145/3098279.3098546.

[16] F. Farbiz, Z. H. Yu, C. Manders, and W. Ahmad, "An electrical muscle stimulation haptic feedback for mixed reality tennis game," in ACM SIGGRAPH 2007 posters, New York, NY, USA, Aug. 2007, pp. 140-es, doi: 10.1145/1280720.1280873.

[17] P. E. Fortin, J. R. Blum, and J. R. Cooperstock, "Raising the Heat: Electrical Muscle Stimulation for Simulated Heat Withdrawal Response," in Adjunct Publication of the 30th Annual ACM Symposium on User Interface Software and Technology, New York, NY, USA, Oct. 2017, pp. 137-139, doi: 10.1145/3131785.3131828.

[18] E. Grönvall, J. Fritsch, and A. Vallgårda, "FeltRadio: Sensing and Making Sense of Wireless Traffic," in Proceedings of the 2016 ACM Conference on Designing Interactive Systems - DIS '16, Brisbane, QLD, Australia, 2016, pp. 829-840, doi: $10.1145 / 2901790.2901818$.

[19] K. Gui and D. Zhang, "Influence of volitional contraction on muscle response to functional electrical stimulation," in 2014 IEEE 19th International Functional Electrical Stimulation Society Annual Conference (IFESS), Sep. 2014, pp. 1-4, doi: 10.1109/IFESS.2014.7036738

[20] S. Hanagata and Y. Kakehi, "Paralogue: A Remote Conversation System Using a Hand Avatar which Postures are Controlled with Electrical Muscle Stimulation," in Proceedings of the 9th Augmented Human International Conference, New York, NY, USA, Feb. 2018, pp. 1-3, doi: 10.1145/3174910.3174951.

[21] M. Hassib, M. Pfeiffer, S. Schneegass, M. Rohs, and F. Alt, "Emotion Actuator: Embodied Emotional Feedback through Electroencephalography and Electrical Muscle Stimulation," in Proceedings of the 2017 CHI Conference on Human Factors in Computing Systems, New York, NY, USA, May 2017, pp. 6133-6146, doi: 10.1145/3025453.3025953.

[22] T. Ishimaru and S. Saga, "Virtual bumps display based on electrical muscle stimulation," in 2020 IEEE Haptics Symposium (HAPTICS), Mar. 2020, pp. 96-101, doi: 10.1109/HAPTICS45997.2020.ras.HAP20.17.61243dc4.

[23] S. Jain, S. Sharma, and D. Babbar, "Star-Force: A Playful Implementation of the Jedi-force," in Proceedings of the Tenth International Conference on Tangible, Embedded, and Embodied Interaction - TEI '17, Yokohama, Japan, 2017, pp. 761-766, doi: $10.1145 / 3024969.3041098$

[24] S. Kasahara, J. Nishida, and P. Lopes, "Preemptive Action: Accelerating Human Reaction using Electrical Muscle Stimulation Without Compromising Agency," in Proceedings of the 2019 CHI Conference on Human Factors in Computing Systems, New York, NY, USA, May 2019, pp. 1-15, doi: 10.1145/3290605.3300873.

[25] M. Katoh et al., "Optimal selection of electrodes for muscle electrical stimulation using twitching motion measurement," in Proceedings of the 4th Augmented Human International Conference on - AH '13, Stuttgart, Germany, 2013, pp. 237238, doi: $10.1145 / 2459236.2459279$.

[26] O. B. Kaul, M. Pfeiffer, and M. Rohs, "Follow the Force: Steering the Index Finger towards Targets using EMS," in Proceedings of the 2016 CHI Conference Extended Abstracts on Human Factors in Computing Systems, New York, NY, USA, May 2016, pp. 2526-2532, doi: 10.1145/2851581.2892352.

[27] T. Kitamura, H. Mizoguchi, N. Mizukami, S. Sakaino, and T. Tsuji, "Chattering reduction of functional electrical stimulation with the smith compensator," in IECON 2017 - 43rd Annual Conference of the IEEE Industrial Electronics Society, Oct. 2017, pp. 7577-7582, doi: 10.1109/IECON.2017.8217328.

[28] J. Knibbe, P. Strohmeier, S. Boring, and K. Hornbæk, "Automatic Calibration of High Density Electric Muscle Stimulation," Proc. ACM Interact. Mob. Wearable Ubiquitous Technol., vol. 1, no. 3, pp. 1-17, Sep. 2017, doi: 10.1145/3130933.

[29] M. Kono, Y. Ishiguro, T. Miyaki, and J. Rekimoto, "Design and Study of a MultiChannel Electrical Muscle Stimulation Toolkit for Human Augmentation," in 
Proceedings of the 9th Augmented Human International Conference, New York, NY, USA, Feb. 2018, pp. 1-8, doi: 10.1145/3174910.3174913.

[30] M. Kono, T. Takahashi, H. Nakamura, T. Miyaki, and J. Rekimoto, "Design Guideline for Developing Safe Systems that Apply Electricity to the Human Body," ACM Trans. Comput.-Hum. Interact., vol. 25, no. 3, p. 19:1-19:36, Jun. 2018, doi: $10.1145 / 3184743$.

[31] Y. Kurita, T. Ishikawa, and T. Tsuji, "Stiffness Display by Muscle Contraction Via Electric Muscle Stimulation,” IEEE Robot. Autom. Lett., vol. 1, no. 2, pp. 1014-1019, Jul. 2016, doi: 10.1109/LRA.2016.2529689.

[32] P. Lopes, S. You, A. Ion, and P. Baudisch, "Adding Force Feedback to Mixed Reality Experiences and Games using Electrical Muscle Stimulation," in Proceedings of the 2018 CHI Conference on Human Factors in Computing Systems - CHI '18, Montreal QC, Canada, 2018, pp. 1-13, doi: 10.1145/3173574.3174020.

[33] P. Lopes, S. You, L.-P. Cheng, S. Marwecki, and P. Baudisch, "Providing Haptics to Walls \& Heavy Objects in Virtual Reality by Means of Electrical Muscle Stimulation," in Proceedings of the 2017 CHI Conference on Human Factors in Computing Systems, Denver, Colorado, USA, May 2017, pp. 1471-1482, doi 10.1145/3025453.3025600.

[34] P. Lopes and P. Baudisch, "Muscle-propelled force feedback: bringing force feedback to mobile devices," in Proceedings of the SIGCHI Conference on Human Factors in Computing Systems, New York, NY, USA, Apr. 2013, pp. 2577-2580, doi $10.1145 / 2470654.2481355$.

[35] Z. Lou, P. Yao, and D. Zhang, "Wireless Master-Slave FES Rehabilitation System Using sEMG Control," in Intelligent Robotics and Applications, Berlin, Heidelberg, 2012, pp. 1-10, doi: 10.1007/978-3-642-33515-0_1.

[36] Y. Nagashima, "Bio-sensing systems and bio-feedback systems for interactive media arts," in Proceedings of the 2003 conference on New interfaces for musical expression, SGP, May 2003, pp. 48-53, Accessed: Sep. 16, 2020. [Online].

[37] J. Nishida, K. Takahashi, and K. Suzuki, "A wearable stimulation device for sharing and augmenting kinesthetic feedback," in Proceedings of the 6th Augmented Human International Conference, New York, NY, USA, Mar. 2015, pp. 211-212, doi: $10.1145 / 2735711.2735775$.

[38] J. Nishida, S. Kasahara, and K. Suzuki, "Wired muscle: generating faster kinesthetic reaction by inter-personally connecting muscles," in ACM SIGGRAPH 2017 Emerging Technologies, New York, NY, USA, Jul. 2017, pp. 1-2, doi $10.1145 / 3084822.3084844$

[39] J. Nishida and K. Suzuki, "bioSync: A Paired Wearable Device for Blending Kinesthetic Experience," in Proceedings of the 2017 CHI Conference on Human Factors in Computing Systems, New York, NY, USA, May 2017, pp. 3316-3327, doi: $10.1145 / 3025453.3025829$.

[40] M. Pfeiffer, T. Duente, and M. Rohs, "A Wearable Force Feedback Toolkit with Electrical Muscle Stimulation," in Proceedings of the 2016 CHI Conference Extended Abstracts on Human Factors in Computing Systems - CHI EA '16, San Jose, California, USA, 2016, pp. 3758-3761, doi: 10.1145/2851581.2890238.

[41] M. Pfeiffer, S. Schneegass, F. Alt, and M. Rohs, "Let me grab this: a comparison of EMS and vibration for haptic feedback in free-hand interaction," in Proceedings of the 5th Augmented Human International Conference, New York, NY, USA, Mar 2014, pp. 1-8, doi: 10.1145/2582051.2582099.

[42] M. Pfeiffer, S. Schneegaß, and F. Alt, "Supporting interaction in public space with electrical muscle stimulation," in Proceedings of the 2013 ACM conference on Pervasive and ubiquitous computing adjunct publication, New York, NY, USA, Sep. 2013, pp. 5-8, doi: 10.1145/2494091.2494094.

[43] M. Pfeiffer and W. Stuerzlinger, "3D Virtual Hand Selection with EMS and Vibration Feedback," in Proceedings of the 33rd Annual ACM Conference Extended Abstracts on Human Factors in Computing Systems, New York, NY, USA, Apr. 2015, pp. 1361-1366, doi: 10.1145/2702613.2732763.

[44] H. Pohl, K. Hornbæk, and J. Knibbe, "Wanding Through Space: Interactive Calibration for Electric Muscle Stimulation," in Proceedings of the 9th Augmented Human International Conference on - AH '18, Seoul, Republic of Korea, 2018, pp 1-5, doi: 10.1145/3174910.3174948.

[45] S. Schneegass, A. Schmidt, and M. Pfeiffer, "Creating user interfaces with electrical muscle stimulation," Interactions, vol. 24, no. 1, pp. 74-77, Dec. 2016, doi $10.1145 / 3019606$.

[46] S. Schneegass and R. Rzayev, "Embodied notifications: implicit notifications through electrical muscle stimulation," in Proceedings of the 18th International
Conference on Human-Computer Interaction with Mobile Devices and Services Adjunct, New York, NY, USA, Sep. 2016, pp. 954-959, doi: 10.1145/2957265.2962663.

[47] T. Shao, X. Li, H. Yokoi, and D. Zhang, "FESleeve: A Functional Electrical Stimulation System with Multi-electrode Array for Finger Motion Control," in Intelligent Robotics and Applications, Cham, 2016, pp. 191-199, doi: 10.1007/978-3-319-435183_19.

[48] E. Tamaki, T. Chan, and K. Iwasaki, "UnlimitedHand: Input and Output Hand Gestures with Less Calibration Time", in Proceedings of the 29th Annual Symposium on User Interface Software and Technology, New York, NY, USA, Oct. 2016, pp. $163-165$, doi: 10.1145/2984751.2985743.

[49] S. Tatsuno, T. Hayakawa, and M. Ishikawa, "Comparison of reaction times in response to electrical and Visual Stimulation using a high-speed camera," in 2016 IEEE International Conference on Systems, Man, and Cybernetics (SMC), Oct. 2016, pp. 001251-001256, doi: 10.1109/SMC.2016.7844413.

[50] S. Tatsuno, T. Hayakawa, and M. Ishikawa, "Supportive training system for sports skill acquisition based on electrical stimulation," in 2017 IEEE World Haptics Conference (WHC), Jun. 2017, pp. 466-471, doi: 10.1109/WHC.2017.7989946.

[51] S. Tatsuno, T. Hayakawa, and M. Ishikawa, "Trajectory adjustment system for learning based on electrical stimulation," in Proceedings of the 8th Augmented Human International Conference, New York, NY, USA, Mar. 2017, pp. 1-4, doi: $10.1145 / 3041164.3041197$

[52] K. Watanabe, M. Oka, and H. Mori, "Feedback Control to Target Joints Angle in Middle Finger PIP and MP Joint Using Functional Electrical Stimulation," Lect. Notes Comput. Sci. Subser. Lect. Notes Artif. Intell. Lect. Notes Bioinforma., vol. 11570 LNCS, pp. 440-454, 2019, doi: 10.1007/978-3-030-22649-7_35.

[53] T. Watanabe, K. Iibuchi, K. Kurosawa, and N. Hoshimiya, "A method of multichannel PID control of two-degree-of-freedom wrist joint movements by functional electrical stimulation," Syst. Comput. Jpn., vol. 34, no. 5, pp. 25-36, 2003, doi: 10.1002/scj.10298.

[54] A. Popović-Bijelić, G. Bijelić, N. Jorgovanović, D. Bojanić, M. B. Popović, and D. B. Popović, "Multi-field surface electrode for selective electrical stimulation," Artif. Organs, vol. 29, no. 6, pp. 448-452, Jun. 2005, doi: 10.1111/j.1525-1594.2005.29075.x.

[55] J. Limanowski, P. Lopes, J. Keck, P. Baudisch, K. Friston, and F. Blankenburg, "Action-Dependent Processing of Touch in the Human Parietal Operculum and Posterior Insula," Cereb. Cortex, vol. 30, no. 2, pp. 607-617, Mar. 2020, doi: 10.1093/cercor/bhz111.

[56] "Haptic Workstation," CyberGlove Systems LLC. http://www.cyberglovesystems. com/haptic-workstation (accessed Sep. 16, 2020).

[57] "CyberGrasp," CyberGlove Systems LLC. http://www.cyberglovesystems.com/ cybergrasp (accessed Sep. 16, 2020).

[58] "Dexta Robotics - Touch the Untouchable." https://origin.dextarobotics.com/enus/ (accessed Sep. 16, 2020).

[59] P. Strojnik, A. Kralj, and I. Ursic, "Programmed Six-Channel Electrical Stimulator for Complex Stimulation of Leg Muscles During Walking," IEEE Trans. Biomed. Eng., 1979, doi: 10.1109/TBME.1979.326520.

[60] E. Kruijff, D. Schmalstieg, and S. Beckhaus, "Using neuromuscular electrical stimulation for pseudo-haptic feedback," in Proceedings of the ACM symposium on Virtual reality software and technology, New York, NY, USA, Nov. 2006, pp. 316-319, doi: 10.1145/1180495.1180558.

[61] P. Lopes and P. Baudisch, "Immense Power in a Tiny Package: Wearables Based on Electrical Muscle Stimulation," IEEE Pervasive Comput., vol. 16, no. 3, pp. 12-16, 2017, doi: 10.1109/MPRV.2017.2940953.

[62] J. de Kroon, M. J. Ijzerman, J. Chae, G. J. Lankhorst, and G. Zilvold, Relation between stimulation characteristics and clinical outcome in studies using electrical stimulation to improve motor control of the upper extremity in stroke. Centre for Reviews and Dissemination (UK), 2005.

[63] M. Schünke, E. Schulte, U. Schumacher, and E. D. Lamperti, Thieme Atlas of Anatomy: Latin Nomenclature: General Anatomy and Musculoskeletal System. Thieme, 2006.

[64] C. E. Lang and M. H. Schieber, "Human Finger Independence: Limitations due to Passive Mechanical Coupling Versus Active Neuromuscular Control," f. Neurophysiol., vol. 92, no. 5, pp. 2802-2810, Nov. 2004, doi: 10.1152/jn.00480.2004.

[65] H. Kajimoto, "Electrotactile Display with Real-Time Impedance Feedback Using Pulse Width Modulation," IEEE Trans. Haptics, vol. 5, no. 2, pp. 184-188, Apr. 2012, doi: 10.1109/TOH.2011.39. 